\title{
Characterizing the Flux of Atmospheric Neutrinos with IceCube-DeepCore
}

\author{
The IceCube Collaboration ${ }^{1}$ \\ ${ }^{1}$ http://icecube.wisc.edu/collaboration/authors/icrc17_icecube \\ E-mail: trwoodeualberta.ca
}

\begin{abstract}
The IceCube Neutrino Observatory instruments more than a cubic-kilometre of the deep glacial ice below South Pole Station, Antarctica, creating the world's largest water Cherenkov detector. With the addition of a low-energy detection array, DeepCore, completed in 2010, the observatory is sensitive to neutrinos with energies between $\sim 5 \mathrm{GeV}$ and the $\mathrm{EeV}$-scale. IceCube has now accumulated the world's largest sample of atmospheric neutrinos, providing the ability to perform precision studies of the flux over the full energy range of the detector. We present sensitivities of atmospheric neutrino flux measurements from $\sim 6 \mathrm{GeV}-180 \mathrm{GeV}$ with particular attention to the kaon-to-pion ratio. This analysis will fill in the overlapping regions of atmospheric neutrino flux measurements established at low energies by Super-Kamiokande and at higher energies by IceCube, bridging the current experimental results.
\end{abstract}

Corresponding author: Tania Wood

Department of Physics, University of Alberta, Edmonton, AB, Canada T6G $2 G 7$

35th International Cosmic Ray Conference ICRC2017-

10-20 July, 2017

Bexco, Busan, Korea 


\section{Introduction}

Precision measurements of atmospheric neutrinos provide an opportunity to study neutrino interactions with world-leading sensitivity. The atmospheric neutrino spectrum provides a sample that has been used to study neutrino oscillations very successfully $[1,2]$. In addition, due to the advancement of neutrino detectors, atmospheric neutrino measurements are now capable of providing precision input to cosmic ray and hadronic interaction models. Atmospheric neutrino flux calculations [3,4] require as minimum input: the cosmic ray spectrum model (which provides the energy spectrum of the incident particles that create the particle shower), the hadronic interaction model (which governs the production of particles as well as their interactions) and the atmospheric density profile model (which provides the atmospheric content, thickness and particle density). The cosmic ray spectrum model and hadronic interaction model represent the largest uncertainties in these predictions [5]; their precision study remains a challenge for the field.

IceCube's DeepCore sub-array [6] measures atmospheric neutrinos in an energy range suited for addressing these questions. The unprecedented statistics of neutrino candidates collected by the DeepCore detector make it possible to study the spectral shape of atmospheric neutrinos, which is closely tied to that of the cosmic rays. Moreover, the detector is sensitive to the crossover region between the dominant meson production in the showers, pions and kaons, which are in turn tied to the hadronic interactions. Here we present an analysis of the atmospheric neutrino spectrum, as well as a sensitivity to the relative contribution of kaons and pions to the neutrino flux using a sample collected by IceCube-DeepCore.

\section{Detector Description}

The IceCube Neutrino Observatory [7] is a large-scale Cherenkov detector that uses the West Antarctic Ice Sheet as the detection medium for charged particles produced in neutrino interactions. The natural deep ice, reaching nearly $3 \mathrm{~km}$ thickness at the Amundsen-Scott South Pole Station, is an excellent Cherenkov medium due to its high optical clarity [8]. The IceCube detector array instruments more than a cubic kilometer of the ice, at depths between approximately $1.5 \mathrm{~km}$ and $2.5 \mathrm{~km}$. In total, 5160 digital optical modules (DOMs) [7], consisting of a 10-inch photomultiplier tube and full on-board data acquisition contained in a glass pressure housing, are deployed on a hexagonal grid of 86 strings, with 60 DOMS per string.

The primary IceCube array consists of 78 strings with $125 \mathrm{~m}$ spacing between strings and DOM-to-DOM spacing of $17 \mathrm{~m}$. In the analysis presented here, this high-energy portion of the detector is used as an active veto region to reject atmospheric muon events [9]. The remaining 8 strings make up the DeepCore sub-array, instrumenting approximately $10^{7} \mathrm{~m}^{3}$ of the ice in the bottom-center of the IceCube array [6]. This is the deepest, clearest ice of the instrumented region [8] and, with a string spacing between $40 \mathrm{~m}$ and $70 \mathrm{~m}$, and a DOM-to-DOM spacing of $7 \mathrm{~m}$, provides a low-energy threshold for neutrino interactions of $\sim 5 \mathrm{GeV}$. The DeepCore data set is the primary source for the analysis presented here, and events typically have a pointing resolution of $\sim 25^{\circ}$ at $30 \mathrm{GeV}$. 


\section{Neutrino Flux Modeling}

Cosmic rays incident on the Earth interact with nuclei in the atmosphere, resulting in hadronic showers that produce a multitude of secondary particles [3]. The particles include mesons that then ultimately decay into charged leptons and neutrinos.

The energy spectra and zenith distribution of these leptons contain information about the primary cosmic rays, hadronic interactions and the decay chain leading to their production. Figure 1 shows the relative contributions of meson decays to the atmospheric neutrino flux in the energy regime for the analysis. As shown in Figure 1, the $v_{\mu}$ flux is dominated by contributions arising from the decay of charged pions $\left(\pi^{ \pm}\right)$and kaons $\left(K^{ \pm}\right)$. Over the energy range of the sample, the dominant contributing mesons are pions at lower energies with kaons becoming dominant above $\sim 80 \mathrm{GeV}$, depending on the zenith angle and choice of hadronic interaction model. For $v_{e}$, the flux is dominated by contributions from the decay of muons, $K_{L}^{0}$ and $K^{ \pm}$.

The kaon-to-pion ratio $(\mathrm{K} / \pi)$ is used in parametrizations of the muon neutrino $\left(v_{\mu}\right)$ flux and is defined as the quotient of the fraction of leptons coming from kaon decay over the fraction of leptons coming from pion decay. It depends on branching ratios and energy distributions of a given decay and is a function of zenith. A good knowledge of these processes is needed to obtain a robust understanding of the spectrum at higher energies and associated uncertainties. This knowledge is critical for atmospheric neutrino studies of fundamental neutrino properties and to determine backgrounds for astrophysical neutrino searches.

As shown in Figure 2, the atmospheric muon neutrino flux produced by $\pi$ has a notable zenith and energy dependence. This leads to a zenith and energy dependence for the $\mathrm{K} / \pi$ ratio. The zenith angle dependence derives from the variation of the atmosphere traversed by the mesons and the difference in their lifetimes. The angular dependence of the $\pi$ component creates zenith dependent rate changes observable by IceCube, making this analysis sensitive to the $\mathrm{K} / \pi$ ratio. The sensitivity to the $\mathrm{K} / \pi$ ratio is important as a calibration input to interaction models and for the benchmarking of flux models.

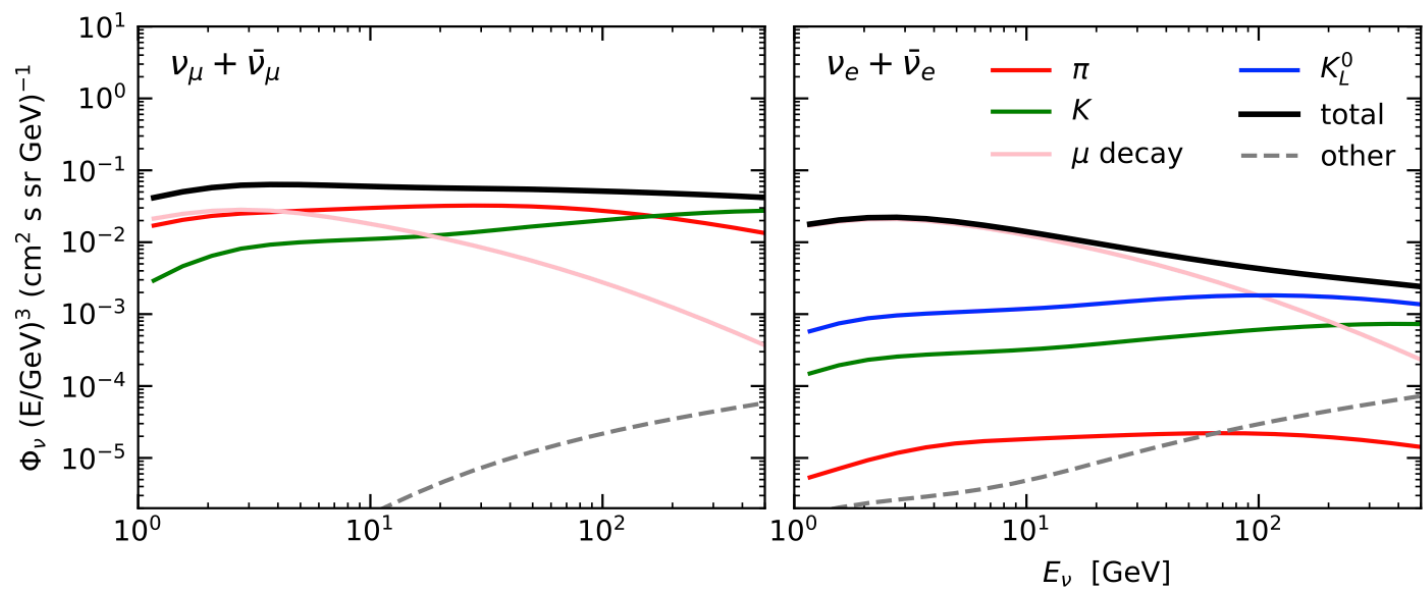

Figure 1: Partial contribution of intermediate particles to the flux of atmospheric neutrinos, muon neutrinos (left) and electron neutrinos (right) at zenith angle of $60^{\circ}$. The primary (Cosmic Ray) spectrum is GaisserHonda [4] and the interaction model is DPMJET-III [21, 22]. This plot is created with MCEq [10]. 

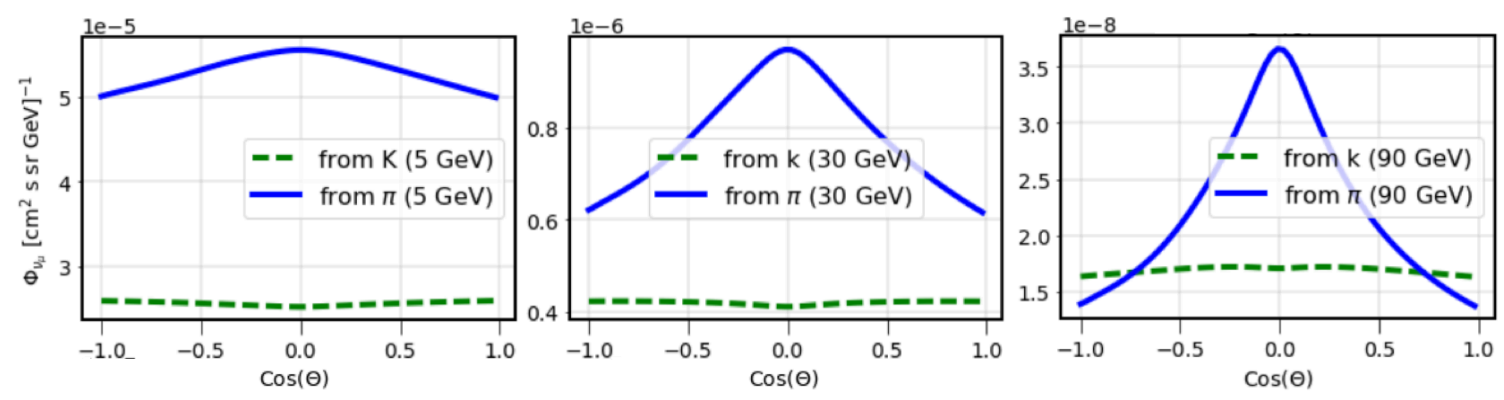

Figure 2: Atmospheric muon neutrino flux dependence on zenith-angle and energy, here shown at $5 \mathrm{GeV}$, $30 \mathrm{GeV}$ and $90 \mathrm{GeV}$. The zenith angle shown is relative to the IceCube detector coordinate system, where -1 corresponds to 'upgoing events'.

\subsection{Current uncertainties on the atmospheric neutrino flux}

For neutrinos at $\mathrm{GeV}$ energies, the availability of secondary particle production from accelerators and direct cosmic ray flux measurements, results in good model predictions [5]. At higher energies the hadronic and primary flux uncertainties in the absolute neutrino flux increase $[5,11]$. As kaons become more relevant as mother meson above approximately $\sim 70 \mathrm{GeV}$ neutrino energy, the largest error on the high energy neutrino fluxes comes from the modeling of kaon production. The absence of fixed-target kaon measurements on light nuclear targets results drives the extrapolation errors of hadronic interaction models. For $\mathrm{TeV}$ neutrinos, cosmic ray observations in the relevant range become indirect and errors from the primary flux model contribute significantly to the total uncertainty.

The current state of the art characterization of uncertainties is the study of Ref. [5]. The authors assign an uncertainty estimation to each region of the energy-momentum fraction phasespace based on their evaluation of globally available fixed-target data. Due to the steep primary spectrum, the relevant phase-space for inclusive lepton production is $x_{\mathrm{F}} \gtrsim 0.2$ [16], where $x_{\mathrm{F}}$ is feynman-x or longitudinal momentum fraction [17]. There are very few measurements of $x_{\mathrm{F}} \gtrsim 0.2$ as this is the very forward $p_{t}$ (transverse momentum) region extremely close to the beam, which accelerator based experiments do not currently measure. There are limited fixed target experiments that do cover some of this region and their integration into these models would be a next step. Atmospheric leptons are largely sensitive to $x_{\mathrm{F}} \gtrsim 0.2$, and correspondingly this lack of data in the models leads to large model uncertainties in our region of analysis.

To summarize, current estimates have $\geq 30 \%$ uncertainty in kaon production and $\geq 15 \%$ uncertainty in pion production in the projectile fragmentation region leading to uncertainty in hadronic models in the region that is relevant for the mesons produced in the atmosphere [5]. The $\mathrm{K} / \pi$ ratio measurement outlined could be used to improve and constrain hardronic interaction models in $x_{\mathrm{F}} \gtrsim 0.2$ and will represent significant improvement over current measurements. This can be seen in the profile likelihood shown in Fig 4, which finds a projected sensitivity of $18 \%$ in the $\mathrm{K} / \pi$ ratio at the $1 \sigma$ line (drawn as the horizontal dotted line at 1.0), which one compares to the current best values stated above. 


\subsection{Simulation software}

The program Matrix Cascade Equation (MCEq) [10, 18] is a numerical solver of the discrete form of the cascade equation. It facilitates the exploration of the impact from using various models (cosmic ray flux model, hadronic interaction models, atmosphere/density profiles). By testing all possible combinations and varying their input parameters we can explore uncertainties. There, the $\mathrm{K} / \pi$ ratio can be directly computed for a range of physically well motivated possibilities. In our simulation, we calculated the neutrino fluxes specifically for the IceCube detector location, which determines the parameterization of the atmospheric density profile as a function of zenith, averaged over azimuth.

The cosmic ray primary models considered are Gaisser-Honda (GH) [4] and H3a [24]. The static atmosphere employed here is the NRLMSISE-00 model [19]. The hadronic interaction models are SYBILL2.3 [20], EPOS-LHC [23] and DPMJET-III [22, 21].

\section{Data sample}

The data under consideration was collected by IceCube-DeepCore and was prepared with the goal of studying atmospheric neutrino oscillations [9]. The sample, approximately 15,000 events per year, has a $95 \%$ neutrino purity with the remainder comprised of cosmic ray muons. The sample is dominated by muon neutrino events and we accept these for all directions ('full-sky') in the reconstructed energy range between $\sim 6 \mathrm{GeV}$ to $180 \mathrm{GeV}$, extended from the energy range of $\sim 6 \mathrm{GeV}$ to $56 \mathrm{GeV}$ of the original sample. Most notably, this sample uses improved event reconstruction compared to that reported in [2], allowing for events with less direct light and events that are 'down-going' to be included in the sample [9]. This improvement in reconstruction was achieved by performing a likelihood-based reconstruction of all events that accounted for both photon scattering and the large-scale variations in the naturally formed glacial ice medium. The inclusion of the down-going events is particularly beneficial since they are primarily unoscillated and therefore help to constrain a source of systematic uncertainty. Backgrounds in the sample from cosmic ray muons are estimated directly from the data, since sufficient simulation is not feasible.

\section{Analysis Method and Sensitivity Estimates}

Data used in the development of this analysis are generated from Monte Carlo (MC) simulation corresponding to the observed events of the described sample from the completed IceCube detector configuration with all high level cuts applied. Sensitivity to a given parameter is explored with Asimov tests using data generated in the same manner. This test data and the MC set are compared using a $\chi^{2}$, described in [9]. The systematic uncertainties related to the flux of neutrinos, as well as those related to the detector, are parameterized and included as nuisance parameters to the fit, with any prior knowledge for a given parameter added to the $\chi^{2}$.

\section{$5.1 \mathrm{~K} / \pi$ ratio fits}

As illustrated in Figure 2, the atmospheric muon neutrino flux is a function of energy and direction. MCEq is used to create tables that contains the expected neutrino flux for each zenith 

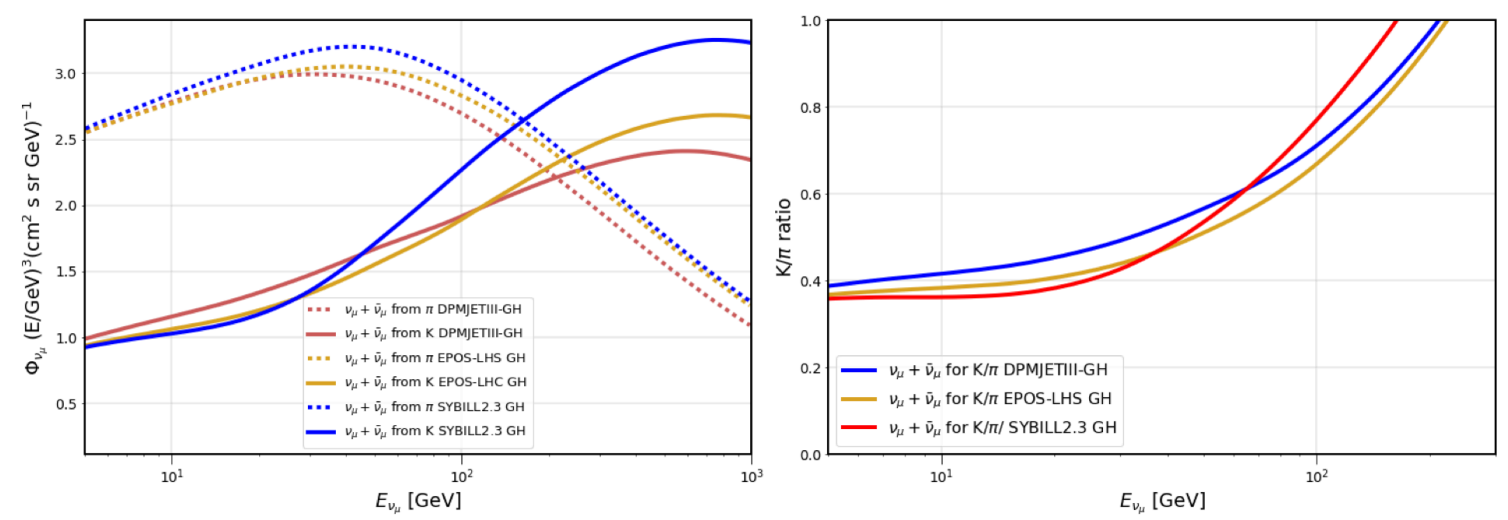

Figure 3: Left: The contributions of pions and kaons to the muon neutrino flux integrated over zenith angles are shown separately as a function of neutrino energy. Hadronic interaction models included in the plot are: EPOS-LHC [23], DPMJET-III [21, 22], SYBILL2.3 [20]. Right: Integrated atmospheric $v_{\mu}$ flux partial contributions, arising from $\pi$ and K decays. The cosmic ray primary model used was Gaisser-Honda [4] and the atmospheric model is NRLMSISE00 [25]. Plots use [18].

angle and energy. These different tables are then used to weight the available simulation. By comparing the event distributions obtained using different table configurations we estimate of this dataset the sensitivity to the $\mathrm{K} / \pi$ parameter.

In Figure 3 left we see the kaon and pion contributions to the atmospheric muon neutrino flux while changing only the hadronic interaction model. In Figure 3 right we see the muon neutrino flux expected to have kaonic parents over the muon neutrino flux expected to have pionic parents. In this analysis, we keep the template of the atmospheric muon neutrinos decaying from pions fixed and allow the component decaying from kaons to be scaled, and fit this with a $\chi^{2}$ method. The scaling of the pion template allows for shifting of the crossover point where kaons begin to

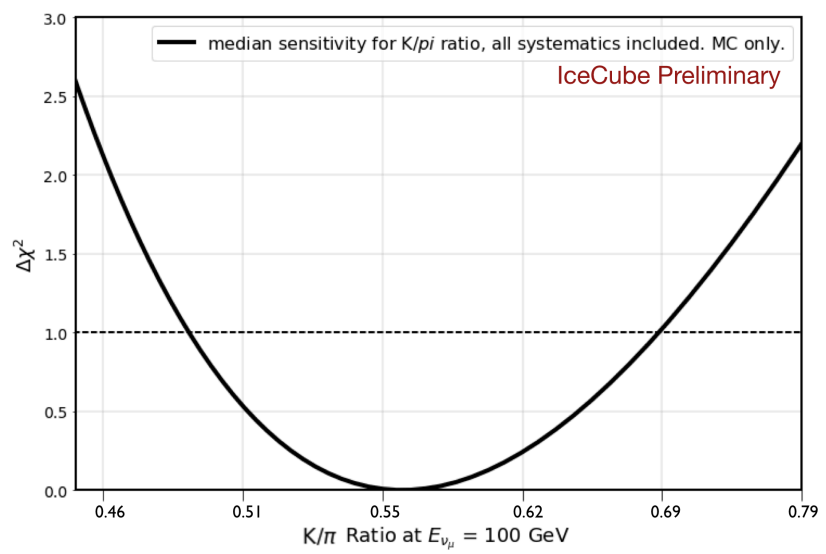

Figure 4: Model independent median sensitivity for IceCube DeepCore to the $\mathrm{K} / \boldsymbol{\pi}$ ratio, all systematics included. Note this plot is an Asimov expectation (no statistical fluctuations). It represents the model independent mean expected sensitivity of our segmented energy spectrum fit to the $\mathrm{K} / \pi$ ratio. The $\mathrm{X}$-axis shows the actual ratio predicted by the model used for zenith information, here DPMJET-III with GH, where 0.55 represents $N_{K}=1$ in Figure 3 . 
dominate as the source for muon neutrinos. The choice of which parent mesons table to fix is arbitrary. The relationship between Figure 3 and Figure 4 can be understood through the following equation: $\Phi=N\left(\Phi_{\pi}+N_{K} \Phi_{k}\right)$, where $\Phi$ represents the flux, $N$ is the overall normalization of the flux and $N_{K}$ is the normalization of the kaonic parent template. Figure 4 represents the preferred value of $N_{K}$ for a given model and can be used to compute the change in the $\mathrm{K} / \pi$ ratio at all energies. Figure 3 left represents the case where $N_{K}=1$, i.e. the ratio predicted by the model. Figure 4 shows the predicted sensitivity for this analysis to the $\mathrm{K} / \pi$ ratio. The $1 \sigma$ range for the currently expected uncertainties on this parameter are approximately an order of magnitude improvement on current best results [5].

\subsection{Energy spectrum fit}

The fitting of the energy spectrum as seen in Figure 5 is performed with a quasi-modelindependent approach. This is performed by fitting a number of distinct spectral segments in energy, which can together approximate the shape of the full spectrum. Each segment is given an energy dependence of $E^{-3}$, as this is not too dissimilar from any of the model predictions over the small domain of each segment. The zenith profile of each segment is taken to be the same as the prediction from the DPMJET-III hadronic model. In the fit each segment's normalization is treated as a separate free parameter, preserving the the zenith profile and the $v / \bar{v}$ ratio. The $\mathrm{K} / \pi$ ratio is also fit in this process. Each segment consists of a pionic flux template and a kaonic flux template whose zenith profits are taken from DPMJET-III and is normalized as outlined in the previous section. There is one shared scaling factor of the kaonic component. The energy spectrum,

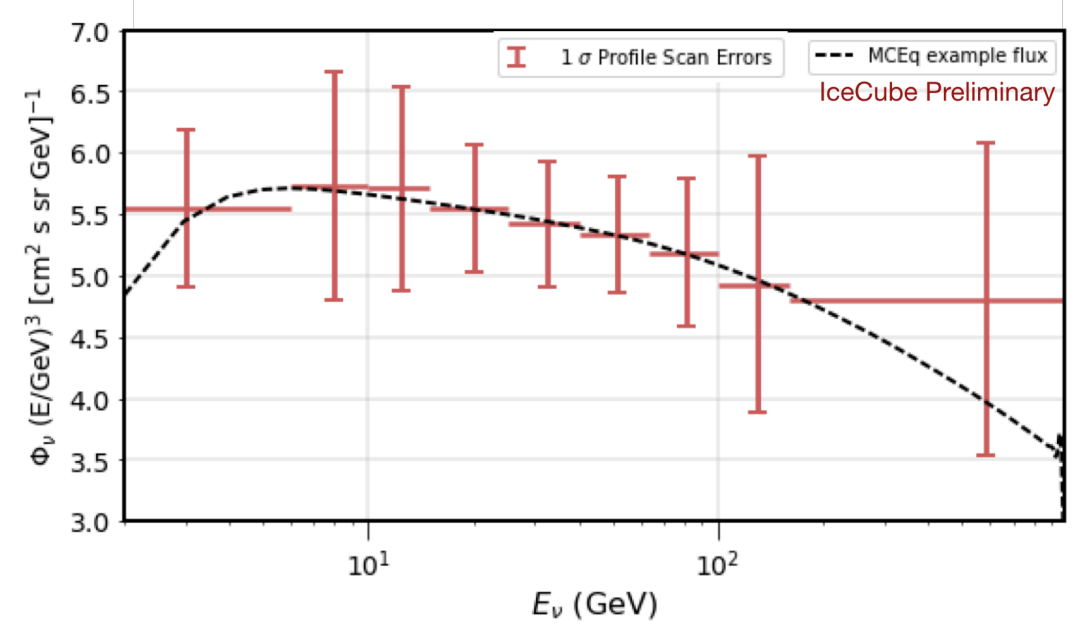

Figure 5: Expected atmospheric muon neutrino flux from MC data, including all systematic and statistical uncertainties for $v_{e}+v_{\mu}$, as a function of the logarithm of the neutrino energy. No statistical fluctuations are included here. The error bars correspond to the $1 \sigma$ band obtained from a profile scan performed for each point. The dashed line shows the model injected as a test spectrum.

oscillation parameters (fitted as nuisance parameters), $\mathrm{K} / \pi$ ratio, and detector systematics are all fit simultaneously. The flux error per point (or segment) is the $1 \sigma$ of a profile scan evaluated for that segment. Preliminary sensitivity using MC only can be seen in Figure 5. 


\section{Future work}

The analysis demonstrates excellent potential for improving our global knowledge on a key energy range and will provide the ability to perform precision studies of the neutrino spectrum over the full energy range of the detector. Going forward, we will split the total atmospheric neutrino energy spectrum into separate $v_{\mu}$ and $v_{e}$ flux components. We anticipate a similar precision to what is shown in Figure 5. The $\mathrm{K} / \pi$ ratio analysis demonstrates the potential to reach precision values and contribute to future hadronic interaction model refinement. Further, the advanced atmospheric neutrino flux modeling tools now in place in this analysis will permit continuing improvement of our treatment of the atmospheric neutrino flux uncertainties, leading to improved oscillation results and neutrino property measurements.

\section{References}

[1] Super-Kamiokande collaboration, Y. Fukuda et. al., PRL 81, 1562 (1998).

[2] IceCube Collaboration, M.G. Aartsen et al., Phys. Rev. D, 91, 072004 (2015).

[3] T. K. Gaisser, R. Engel, and E. Resconi, Cambridge University Press (2016).

[4] T. K. Gaisser and M. Honda, Ann. Rev. Nucl. Part. Sci. 52, 153 (2002).

[5] G. D. Barr, T. K. Gaisser, S. Robbins and T. Stanev, Phys. Rev. D 74094009 (2006).

[6] IceCube Collaboration,R. Abbasi et al., Astropart. Phys. 35, 1109.6096 (2012).

[7] IceCube Collaboration, M. G. Aartsen et al., JINST 12 P03012 (2017).

[8] IceCube Collaboration, M.G. Aartsen et al., Journal of Glaciology 59 (2013).

[9] J.P.A.M Andre for the IceCube Collaboration, IPA Symposium (2017).

[10] A. Fedynitch, R. Engel, T. K. Gaisser, F. Riehn and T. Stanev, PoS ( ICRC2015) 1129 (2016).

[11] A. Fedynitch, J. Becker Tjus and P. Desiati, Phys. Rev. D 86114024 (2012).

[12] NA49 Collaboration, Eur. Phys. J. C 49, 897 (2007),73, 2364 (2013).

[13] NA49 Collaboration, C. Alt et al., Eur. Phys. J. C 45, 343 (2006).

[14] NA49 Collaboration,T. Anticic et al., Eur. Phys. J. C 659 (2010).

[15] NA49 Collaboration, T. Anticic et al., Eur. Phys. J. C 68, 1 (2010).

[16] R. M. Ulrich et al., POS (ICRC2015) 407 (2016).

[17] K. Goulianos, FERMILAB-Conf-94/266-E (1996).

[18] A. Fedynitch, R. Engel, T. K. Gaisser, F. Riehn and T. Stanev, EPJ Web Conf. 99, 08001 (2015).

[19] J. M. Picone, A. E. Hedin, D. P. Drob, and A. C. Aikin, J. Geophys. Res., 107 (A12), 1468, (2002).

[20] F. Riehn, R. Engel, A. Fedynitch, T. K. Gaisser and T. Stanev, PoS (ICRC2015) 558 (2016).

[21] S. Roesler, R. Engel and J. Ranft, Monte Carlo (2000).

[22] A. Fedynitch and R. Engel, ICNRM 2014 (2015).

[23] T. Pierog, I. Karpenko, J. M. Katzy, E. Yatsenko and K. Werner, Phys. Rev. C 92, 034906 (2015).

[24] T. K. Gaisser, Astropart. Phys. 35, 801 (2012).

[25] M. Honda et al., Phys. Rev. D 92,023004 (2015). 